\title{
EL PAPEL DE LA CARACTERIZACIÓN MINERALÓGICA Y GEOQUÍMICA EN EL ESTUDIO DE LAS CULTURAS ANTIGUAS: EJEMPLOS DE EXCAVACIONES ESPAÑOLAS EN ORIENTE
}

\author{
Manuel Pozo Rodríguez \\ Universidad Autónoma de Madrid
}

\begin{abstract}
RESUMEN
Los constituyentes inorgánicos de los materiales arqueológicos están formados por minerales y rocas, o bien productos resultantes de su transformación tecnológica. La correcta caracterización de minerales y rocas permite al arqueólogo e historiador obtener una información importante para establecer su origen y uso, pero también la tecnología empleada. La actividad arqueológica facilita, además, conocer la estratigrafía y cronología de los acontecimientos, lo que permite al geólogo establecer las condiciones paleoambientales de su depósito. En el estudio del entorno y de los materiales de los yacimientos adquieren relevancia la Geoarqueología y la Arqueometría. En las siguientes secciones se describe, de forma sintética, qué son los minerales y rocas, cómo se identifican y, finalmente, varios ejemplos de aplicación en yacimientos de Siria y la península arábiga.
\end{abstract}

PALABRAS CLAVE

Geoarqueología, Arqueometría, minerales y rocas, cerámica, metales

\begin{abstract}
The inorganic constituents of archaeological materials consist of minerals and rocks, or products resulting from their technological transformation. The correct characterization of minerals and rocks allows the archaeologist and historian to acquire important information to establish their origin and use, but also the technology applied. Archaeological activity also makes it easier to determine the stratigraphy and chronology of events, allowing the geologist to establish the palaeoenvironmental conditions of the deposit. Geoarchaeology and Archaeometry acquire relevance in the study of the environment and materials of the sites. The following sections describe, in a synthetic way, the minerals and rocks, as they are identified, and finally several examples of application in archaeological sites in Syria and the Arabian Peninsula.
\end{abstract}

\section{KEYWORDS}

Geoarchaeology, Archaeometry, minerals and rocks, ceramics, metals

\section{Introducción}

La importancia del papel jugado por los estudios de carácter geológico en los trabajos de excavación e interpretación arqueológica son incuestionables. En los estudios relacionados con la Historia, la traducción, el análisis y la interpretación de los documentos escritos juega un papel decisivo en la reconstrucción de lo que sucedió en un pasado más o menos remoto. En ocasiones, la ausencia de textos, como sucede durante la Prehistoria, o su carácter incompleto necesita realizar otro tipo de "lecturas", asociadas en este caso a la reconstrucción del entorno físico o a la interpretación de los materiales empleados de forma cotidiana que, como un libro, si se saben leer, pueden aportar una valiosa información desde el punto de vista de la interpretación histórica (Pozo, 1998). La procedencia de los materiales es en la mayor parte de los casos el resultado de la actividad arqueológica, que además permite establecer secuencias estratigráficas y dataciones de los acontecimientos acaecidos en ese lugar. La Geoarqueología y Arqueometría son ramas científicas multidisciplinares que han realizado importantes aportaciones en el estudio de las excavaciones arqueológicas. 


\section{I.1. Geoarqueología y Arqueometría}

Los principios, métodos y técnicas geológicas no son solo aplicables sino necesarios en los estudios arqueológicos (Pozo, 1998). Efectivamente, el empleo de la Geología y sus técnicas en Arqueología se ha incrementado de forma notable en las últimas décadas. En sus aspectos más básicos, North (1938) indica que el arqueólogo debe estar familiarizado con: 1) La interpretación de mapas geológicos. 2) La identificación de estructuras pétreas naturales y realizadas por el hombre. 3) Establecimiento de la geología del lugar y evaluación de la disponibilidad de materias primas minerales. La aplicación de criterios geológicos en Arqueología ha sido tradicionalmente mayor en Europa que en Estados Unidos, lo cual se debe a la utilización temprana por lo europeos de las técnicas estratigráficas en las excavaciones.

En la actualidad la gran variedad de aplicaciones geológicas y de otras ciencias en Arqueología ha dado lugar a la Geoarqueología y la Arqueometría. Actualmente, se entiende por Geoarqueología la aplicación de los conceptos y métodos de las ciencias de la Tierra (geología, geomorfología, hidrología, sedimentología, edafología y exploración geofísica) a los problemas arqueológicos (Canti, 2001). Aporta evidencias sobre el desarrollo, conservación y destrucción de los yacimientos arqueológicos pero también de los cambios ambientales y evolución regional y local del paisaje, incluyendo su impacto en los grupos humanos.

La denominación de la disciplina científica que aborda la investigación geológicoarqueológica ha sufrido discrepancias. Así, el ambientalista Butzer (1982) diferencia entre Geoarqueología (Arqueología asistida por la metodología de las ciencias de la Tierra) y Geología arqueológica (Geología con predisposición a la aplicación arqueológica). Para Gladfelter (1977), la Geoarqueología se basa fundamentalmente en la geomorfología y la petrología sedimentaria, lo que permite la reconstrucción paleoambiental a diversas escalas.

Por su parte, la Arqueometría adquiere un carácter interdisciplinar incluyendo los métodos de datación absoluta (radioisótopos, termoluminiscencia) y los de caracterización de materiales pétreos, metalúrgicos y cerámicos, pero también de los biomateriales (restos óseos, exoesqueletos). Contempla también el tratamiento conservación y restauración de bienes culturales. En su vertiente geológica se trata de un conjunto de técnicas analíticas y de metodologías científicas, empleadas para la datación, identificación de estructuras y materiales, e interpretación de su procedencia y tecnología. Su aplicación es fundamental en aquellos estudios relacionados con la Arqueología, la Historia o el Arte.

\section{I.2. El estudio del yacimiento y su entorno}

Aquí adquiere relevancia la Geomorfología, siendo su principal objetivo la reconstrucción de los ambientes y paisajes originales del yacimiento arqueológico (Pozo, 1998). La base del estudio geomorfológico es la elaboración de un mapa del yacimiento y de sus alrededores. Este mapa aporta información sobre la red de drenaje, geoformas, rasgos tectónicos, naturaleza de las formaciones superficiales (incluidos suelos) y procesos geomorfológicos activos. La información planimétrica obtenida se complementa con el empleo de criterios estratigráficos que permiten interpretar la evolución vertical y lateral del yacimiento.

La importancia del análisis paleoambiental (reconstrucción del paisaje y clima) como complemento idóneo de los objetivos arqueológicos ha sido destacado por Hassam (1985). En este caso además de la geomorfología adquiere relevancia la sedimentología y la estratigrafía, pero también el estudio de paleosuelos y el análisis de polen, fauna y plantas. 
En los últimos años han adquirido relevancia el empleo de técnicas geofísicas que se basan principalmente en las propiedades magnéticas, eléctricas o elásticas de rocas y sedimentos (Figura 1). Los métodos geofísicos no son destructivos y son menos costosos que la prospección mediante catas, siendo de gran ayuda como fase previa a la excavación de yacimientos complejos o de los que solo se dispone de indicios.

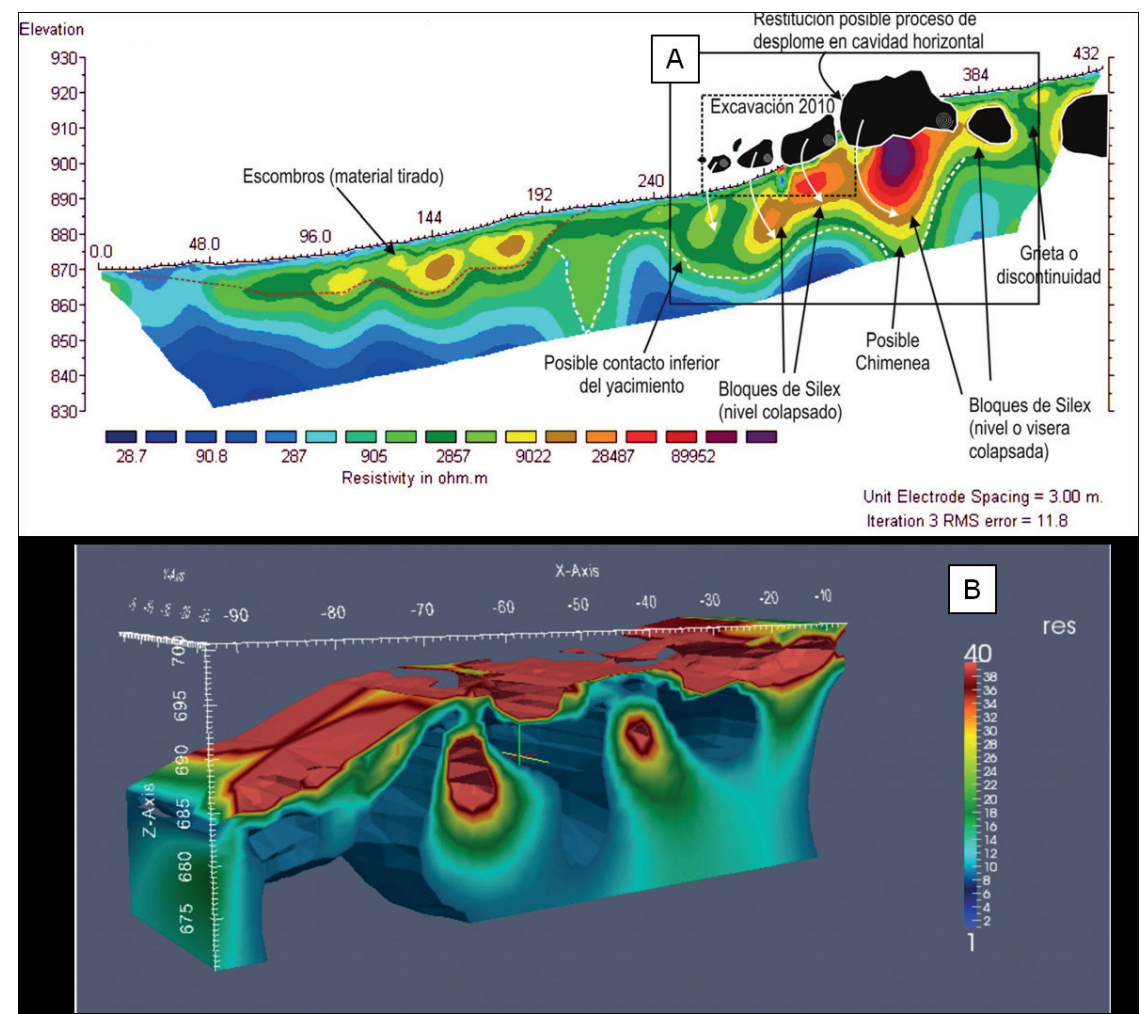

1-Ejemplo de método geofísico. A) Tomografia eléctrica de resistividades (ERT) 2D. B) Tomografía eléctrica de resistividades (ERT) 3D. La variación de resistividad en los diferentes materiales permite reconocer la presencia de cavidades o elementos constructivos en profundidad.

\section{I.3. Estudio de los materiales del yacimiento}

Se puede diferenciar entre datación y caracterización de los materiales. En la datación se emplean técnicas que permiten establecer cronologías, comúnmente incluyen los radioisótopos y la termoluminiscencia. La caracterización mineralógica se basa en técnicas como la difracción de rayos $\mathrm{X}$, que complementada con la petrografía (microscopía óptica y electrónica) permite además el estudio textural de los materiales. Los estudios geoquímicos incluyen el análisis elemental o isotópico. Los análisis químicos de elementos traza han demostrado ser de gran utilidad para el establecimiento de áreas de procedencia, especialmente cuando la información se combina con la mineralogía.

Un caso particular de caracterización de materiales arqueológicos son las cerámicas, en las que se diferencia la pasta arcillosa, el desgrasante y los aditivos superficiales. Desde el punto de vista arqueológico su estudio permite: 1) Caracterizar y clasificar cerámicas fabricadas en regiones geográficas diferentes y/o en épocas distintas. 2) Reconocer las técnicas empleadas en la fabricación de la cerámica, incluyendo las temperaturas alcanzadas en el tratamiento térmico. 3) Identificación de las materias primas empleadas y sus áreas de procedencia.

Los constituyentes inorgánicos de los materiales arqueológicos están constituidos por minerales y rocas, o bien productos resultantes de su transformación tecnológica 
(cerámica, metales). La correcta caracterización de minerales y rocas permite al arqueólogo e historiador obtener una información importante para establecer su origen y uso, pero también la tecnología empleada.

\section{Conceptos básicos sobre minerales y rocas}

Los minerales son el fundamento de nuestro planeta y de nuestra sociedad industrial, son los materiales naturales a partir de los cuales se ha fabricado cualquier producto inorgánico que conocemos. Sin los minerales el hombre no habría podido desarrollar una industria lítica durante la Prehistoria, elaborando herramientas que permitieron la caza, la defensa y otras actividades fundamentales para su subsistencia. El desarrollo de la metalurgia para la extracción de $\mathrm{Cu}$, Sn y Fe habría sido imposible sin los minerales, y lo mismo sería aplicable a la fabricación de las cerámicas. En ambos casos con el empleo de unos conocimientos tecnológicos notables. A lo largo de la historia, las obras civiles se han sustentado básicamente en el empleo de materiales que están formados por minerales, es el caso de edificios, calzadas o acueductos. Llegado este punto se hace necesario entender ¿qué es un mineral? En la actualidad la definición de mineral más completa y ampliamente aceptada es la siguiente: "Un mineral es un compuesto químico sólido y homogéneo, de origen natural, formado como consecuencia de un proceso generalmente inorgánico, dotado de una composición química definida pero no fija y con una estructura interna ordenada".

De los cerca de 4.000 minerales conocidos, sólo unos centenares tienen verdaderamente algún valor económico (menas y minerales industriales) y sólo unos 25 son los componentes principales de la corteza terrestre. Casi todos estos minerales tienen en común el ser silicatos y por lo tanto presentar Si y O como elementos predominantes en su composición. La abundancia de los principales elementos en la corteza terrestre confirma además un quimismo rico en $\mathrm{Al}, \mathrm{Mg}, \mathrm{Fe}, \mathrm{Ca}, \mathrm{Na}$ y $\mathrm{K}$, de manera que éstos ocho elementos representan más del $98 \%$ en peso de la corteza continental del planeta. A pesar de ser mucho menos abundantes que los silicatos (aproximadamente un $8 \%$ de la corteza terrestre) la mayoría del resto de los minerales son importantes desde el punto de vista industrial y económico. Así, la práctica totalidad de los minerales mena de donde se extraen los metales, no son silicatos.

Las rocas son, en la mayoría de los casos, agregados poliminerales, donde sus componentes se disponen con una textura característica del ambiente genético en el que se han formado. Una roca común como el granito está constituida principalmente por tres fases minerales: cuarzo, ortosa y biotita. En algunos casos las rocas pueden ser monominerales, manteniendo rasgos texturales característicos de su ambiente de formación, es el caso del mármol, la cuarcita o el yeso.

El origen y evolución de las rocas se recoge en el denominado ciclo petrológico. En éste se pone de manifiesto como las rocas, y por consiguiente sus minerales constituyentes, se pueden formar en tres ambientes genéticos principales: magmático, metamórfico y sedimentario. Los dos primeros tienen lugar en el interior de la corteza y manto terrestre, por lo que también se denominan endógenos. Por su parte, el ambiente sedimentario se desarrolla sobre la corteza o en zonas relativamente poco profundas de la misma, denominándose también exógeno. Es de destacar que el término exógeno incluye tanto el ambiente sedimentario como el edáfico (suelos).

En un yacimiento arqueológico los materiales pétreos utilizados en la industria lítica, obras civiles u ornamentación son generalmente rocas de diverso origen y características. Por ejemplo, sílex en industria lítica y mármol en la realización de esculturas. En el caso de abalorios y joyas juegan un papel más importante los minerales, como la variscita en cuentas de collar o el rubí en un brazalete. Con respecto a los materiales cerámicos, las 
arcillas y sus minerales de la arcilla constituyentes (silicatos laminares) son fundamentales para entender la capacidad del barro arcilloso para ser moldeado y su endurecimiento tras ser sometido a un tratamiento térmico. Finalmente, los metales, tienen su origen en diversos minerales no silicatados a los que el ser humano en un alarde tecnológico fue capaz de extraer y alear para fabricar útiles y herramientas de cobre, bronce y hierro.

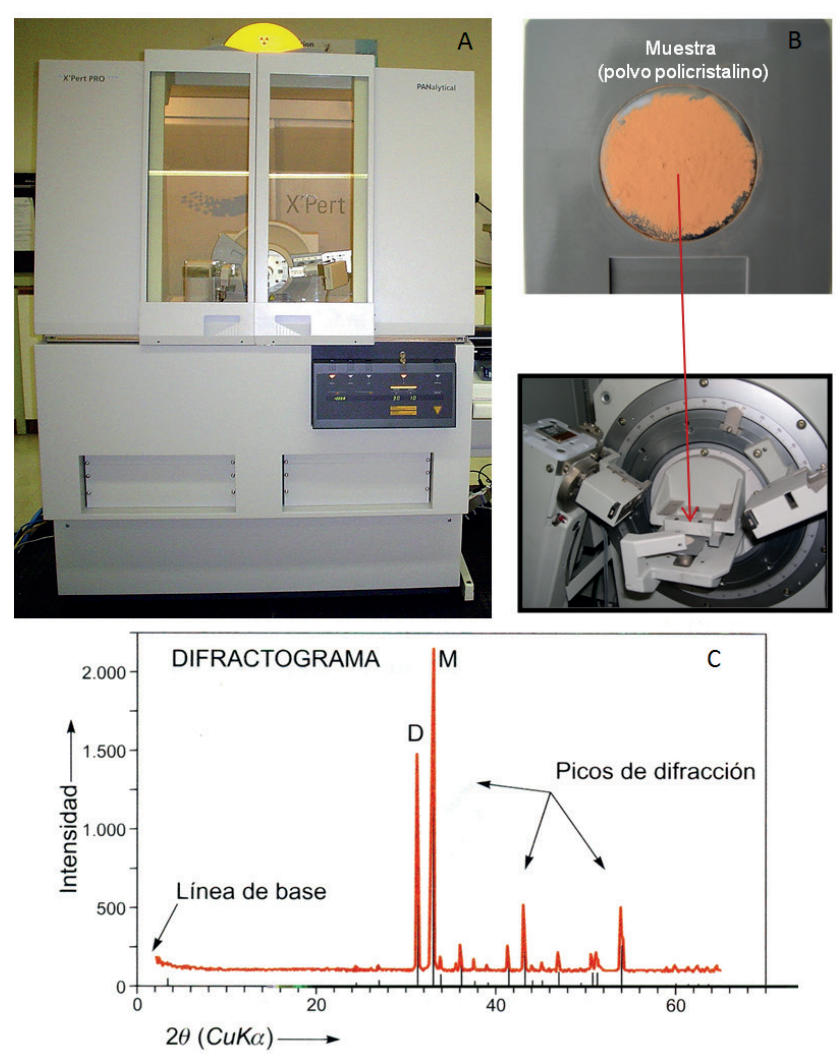

2-Difracción de rayos X (XRD). A) Difractometro de rayos X. B) Muestra de polvo policristalino y ubicación en el difractometro. C) Ejemplo de difractograma mostrando los elementos del mismo, las letras D y M indican los picos más intensos de dos fases presentes.

\section{II.1. Métodos de identificación mineralógica}

Desde antiguo los minerales se han identificado siguiendo criterios de observación visual, principalmente hábito, color y brillo. También mediante la aplicación de un esfuerzo que puede originar rayado (dureza) o rotura (exfoliación y fractura). Complementariamente la estimación del peso específico de un mineral y su solubilidad en medio acuoso o ácido se han empleado como criterios de diagnóstico (Pozo et al., 2004). En la actualidad, las determinaciones anteriormente mencionadas sirven como reconocimiento preliminar, aunque en algunos casos las características del mineral y/o la experiencia del observador puede favorecer su inequívoca identificación. Actualmente existe un gran número de técnicas analíticas para la identificación de minerales, las más comunes son la difracción de rayos X y la petrografía mediante microscopía óptica y electrónica.

La difracción de rayos $\mathrm{X}$ es indudablemente la técnica más ampliamente utilizada en la identificación de minerales. Es también de gran ayuda en la identificación de los constituyentes de una roca, especialmente de los sedimentos y rocas sedimentarias de carácter arcilloso. No obstante, pese a su utilización en el estudio composicional de rocas, de ninguna manera sustituye al análisis petrográfico, sino que lo complementa. El análisis petrográfico permite no solo establecer la composición mineralógica sino además la textura, factor esencial en la caracterización y clasificación de la roca. 


\section{II.1.1. Difracción de rayos $X$}

La difracción de rayos $X$ constituye uno de los métodos más fiable de identificación mineralógica en todos aquellos materiales que presentan una estructura cristalina y por consiguiente sus componentes están ordenados espacialmente. Esta técnica se basa en la interferencia de un haz de rayos X con la red cristalina. Las longitudes de onda empleadas son muy pequeñas (del orden de $1 \AA$ ) y su valor depende del metal empleado para la generación de rayos X; uno de los metales más empleados es el cobre ( $\mathrm{CuK} \alpha=1,5418 \AA$ ).

De las diversas aplicaciones de la difracción de rayos $\mathrm{X}$, la que se emplea con más frecuencia en la identificación de sustancias minerales, es el denominado método del polvo policristalino (Figuras 2A y B). En este método la muestra ha sido finamente pulverizada, por lo que en teoría hay partículas cristalinas con sus planos reticulares en todas las direcciones posibles. En la práctica, la muestra molida a tamaño fino $(<50 \mu \mathrm{m})$ se coloca en un portamuestra y se introduce en un aparato denominado difractómetro de rayos $\mathrm{X}$, donde la muestra se somete a la acción de la radiación que incide de forma continua con distintos ángulos (Pozo et al., 2004). El resultado es un espectro denominado difractograma, donde se recogen una serie de picos que en la escala horizontal muestra el ángulo $2 \theta$ y en la vertical la intensidad del pico difractado (Figura $2 \mathrm{C}$ ). Conociendo la longitud de onda de los rayos $\mathrm{X}(\lambda)$ y el valor del ángulo de difracción $(\theta)$, medido como $2 \theta$ en el difractograma, es muy fácil calcular el valor del espaciado ( $\mathrm{d} \AA$ ); bien mediante aplicación de la ecuación de Bragg ( $n \lambda=2 d$. sen $\theta$ ) o utilizando tablas de conversión de ángulo a espaciado ya preparadas a tal efecto. El conjunto de espaciados y su intensidad es el criterio empleado para la identificación de los minerales constituyentes de la muestra, bien empleando tablas de identificación o mediante el empleo de programas informáticos de análisis mineral. En el caso de que la muestra no presente ordenamiento interno en su estructura (por ejemplo obsidiana), lo que se obtiene es una banda de difracción que nos informa del carácter amorfo del material estudiado.

\section{II.1.2. Petrografia}

El estudio de los minerales y rocas mediante el microscopio de luz polarizada es una de las técnicas más ampliamente utilizada por los geólogos, ya que no solo permite reconocer y cuantificar la composición mineralógica de una muestra, sino también establecer sus características texturales, y por consiguiente inferir su ámbito genético. Aunque es una técnica aplicable a cualquier tipo de material, adquiere especial relevancia en muestras donde los constituyentes mineralógicos son de pequeño tamaño, y en consecuencia difíciles de reconocer a simple vista o con la ayuda de una lupa de bolsillo. El análisis petrográfico de una muestra, de mineral o roca, se sustenta en tres pilares básicos: el microscopio de luz polarizada, las láminas delgadas y las propiedades ópticas de los minerales.

Elmicroscopio petrográfico es en muchos aspectos similaral microscopio convencional denominado biológico. La diferencia más notable es el empleo de luz polarizada para el estudio óptico de los minerales. En un haz de luz normal, no polarizado, las ondas vibran en muchas direcciones diferentes. Sin embargo, es posible filtrar o alterar un haz de luz para hacer que todas las ondas vibren en una dirección paralela a un plano determinado. La luz, se dice entonces que esta polarizada. El empleo de los dos polarizadores orientados con una diferencia de $90^{\circ}$ y la presencia entre ambos de materia cristalina es el fundamento del estudio óptico de los minerales mediante el microscopio petrográfico.

El estudio, identificación y clasificación de minerales y rocas con el microscopio petrográfico se realiza habitualmente en láminas delgadas. En una lámina delgada la muestra original se ha adelgazado hasta alcanzar un grosor de $0,03 \mathrm{~mm}(30 \mu \mathrm{m})$, valor éste que está estandarizado para toda lámina delgada de mineral o roca. Las propiedades ópticas de los minerales sirven como criterio para su identificación, diferenciándose minerales opacos y no 
opacos, y dentro de los segundos minerales isótropos y anisótropos (uniáxicos y biáxicos). Para el examen sintético de las propiedades ópticas de los minerales, se remite al lector al libro "Geología práctica" de Pozo et al. (2004). En materiales metálicos procedentes de estudios de arqueometalurgia se emplea un tipo diferente de microscopio denominado maetalográfico, que funciona con luz reflejada (no transmitida como en el petrográfico) y luz polarizada, en este caso las muestras se presentan en probetas pulidas para su estudio.

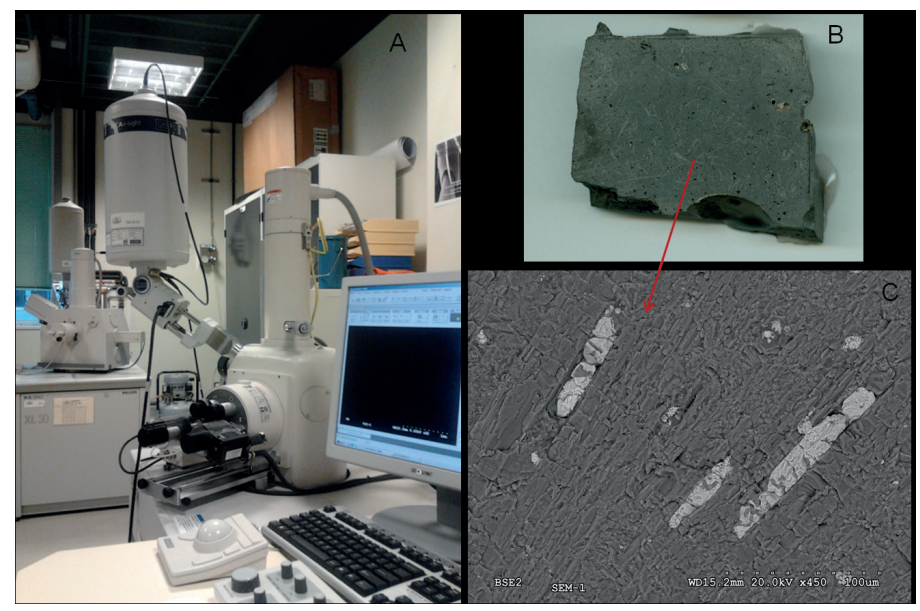

3-Microscopía electrónica de barrido (SEM). Laboratorio mostrando dos microscopios electrónicos con analizador puntual (EDX) incorporado. B) Muestra de escoria procedente de un yacimiento arqueológico. C) Imagen de SEM mostrando la presencia de inclusiones orientadas

de cobre (blanco) en una masa de cristales aciculares de silicatos de hierro (gris oscuro).

Como complemento del estudio petrográfico se emplea con frecuencia la microscopía electrónica de barrido (SEM en inglés). Un haz de electrones sustituye a la luz permitiendo observaciones con mayor resolución a elevados aumentos. Además, incorpora un sistema de análisis puntual que permite conocer la composición química de los constituyentes de la muestra. El pequeño tamaño de las muestras que se pueden examinar la convierte en una técnica idónea en materiales arqueológicos y relacionados con el arte (Figura 3).

\section{Ejemplos de aplicación en yacimientos arqueológicos de Siria y la Península Arabiga}

Con el fin de mostrar la relevancia de las técnicas mineralógicas y petrográficas, expuestas previamente, en los estudios arqueológicos, se recoge en los siguientes apartados varios ejemplos de aplicación a materiales constructivos, pétreos, cerámicos, metálicos $\mathrm{u}$ ornamentales, todos ellos procedentes de yacimientos arqueológicos en Oriente con participación española.

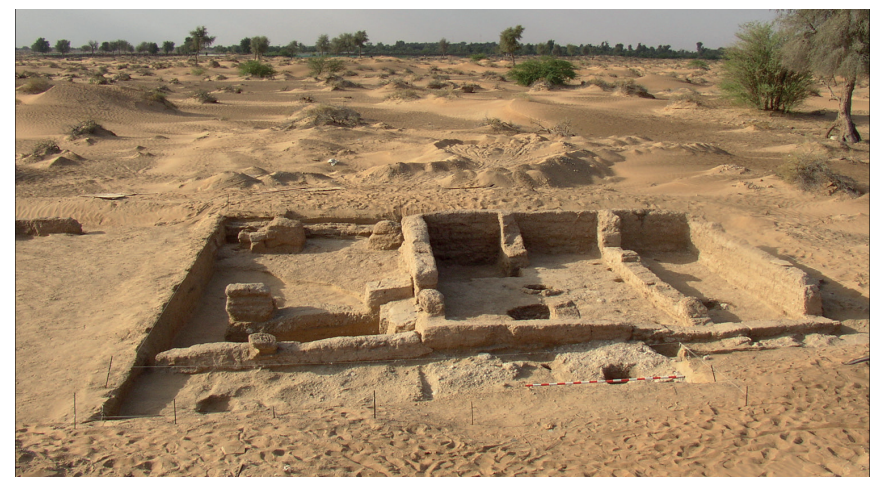

4-Yacimiento arqueológico de Thuqeibah (Emirato de Sharjah). Se observan los muros de una casa del poblado construidos con adobes. 
III.1. El yacimiento de Al-Madam (EAU): elementos constructivos y artefactos

El oasis de al Madam se ubica en el piedemonte de la vertiente oriental de las Montañas de Omán, formando parte de una banda de orientación N-S en la que se distribuyen, los también oasis, de Dhaid, Mleiha y al Ain. Las condiciones favorables de estos enclaves para la vida humana y animal permitieron, desde tiempos muy remotos, el asentamiento de poblados estables en la península de Omán. Las campañas de excavaciones arqueológicas en al Madam han puesto de manifiesto la existencia de un poblado de la Edad del Hierro (1100-300 años a.C.) perfectamente conservado próximo a la localidad de Thuqueibah (AM1) y de una pequeña necrópolis (AM32), no documentada previamente, en la falda oriental del Jabel Buhais. La tecnología de construcción del poblado se caracteriza por el empleo de adobes rectangulares, que se disponen sobre gruesas capas de argamasa, originando muros de gran consistencia (Figura 4).

El estudio analítico de los materiales que constituyen los elementos estructurales de un poblado (muros, suelos, etc.) así como de los artefactos utilizados por sus moradores permite establecer la autoctonía o aloctonía de los materiales empleados (áreas de procedencia). Pero además, se pueden realizar inferencias relativas a su proceso de elaboración. La caracterización textural y composicional (mineralógica y química), de los elementos arqueológicos estructurales y artefactos minerales recogidos durante las excavaciones se describen en diversos trabajos (Pozo y Córdoba, 2002; Pozo et al., 2005). Los materiales estudiados incluyen muestras de adobes quemados y sin quemar, así como muestras del suelo, útiles y fragmentos de vasijas. Las técnicas analíticas empleadas incluyen difracción de rayos $\mathrm{X}$ y petrografía.

\section{Adobes y suelo}

Los adobes presentan una composición caracterizada por el predominio de calcita con dolomita muy subordinada y proporciones variables de cuarzo, feldespatos (predominantemente plagioclasas) y filosilicatos. Otros minerales presentes en contenido variable son piroxeno, olivino y halita. Los adobes con fuerte afectación térmica muestran bajo contenido de filosilicatos y alteración de los granos de carbonato a cal $(\mathrm{CaO}$, portlandita) lo que permite inferir temperaturas entre $650-700^{\circ} \mathrm{C}$. La ausencia de tridimita o de evidencias de fusión incipiente en los bordes de grano del cuarzo implica que los adobes quemados no sufrieron temperaturas superiores a los $800^{\circ} \mathrm{C}$.

En la muestra de suelo estudiada destaca la presencia de rasgos de deformación en poros y de retrabajamiento de clastos. Los componentes del esqueleto parecen disponerse con cierta estructura bandeada, incluyéndose en los mismos fragmentos de adobes, que confirman su papel de asentamiento.

El estudio mineralógico de los elementos estructurales (adobes, suelo) pone de manifiesto una composición similar a la de los materiales del entorno físico, esto es, de los depósitos del wadi próximo, mostrando mezcla de sedimentos fluviales y eólicos.

\section{Útiles pétreos}

Los útiles están constituidos por rocas de diverso tamaño, redondeadas o angulosas, que corresponden a útiles de moler (duras y de tono oscuro) o de articulación de puertas (blandas y de tono claro) (Figura 5). Para la molienda los útiles empleados proceden de rocas ígneas duras y compactas, habiéndose reconocido gabros y peridotitas. El predominio de los primeros estaría en relación con el mayor grado de fracturación y alteración que presentan las peridotitas. En el caso de útiles para la articulación de puertas el material elegido ha sido la caliza, más blanda y fácil de perforar. 


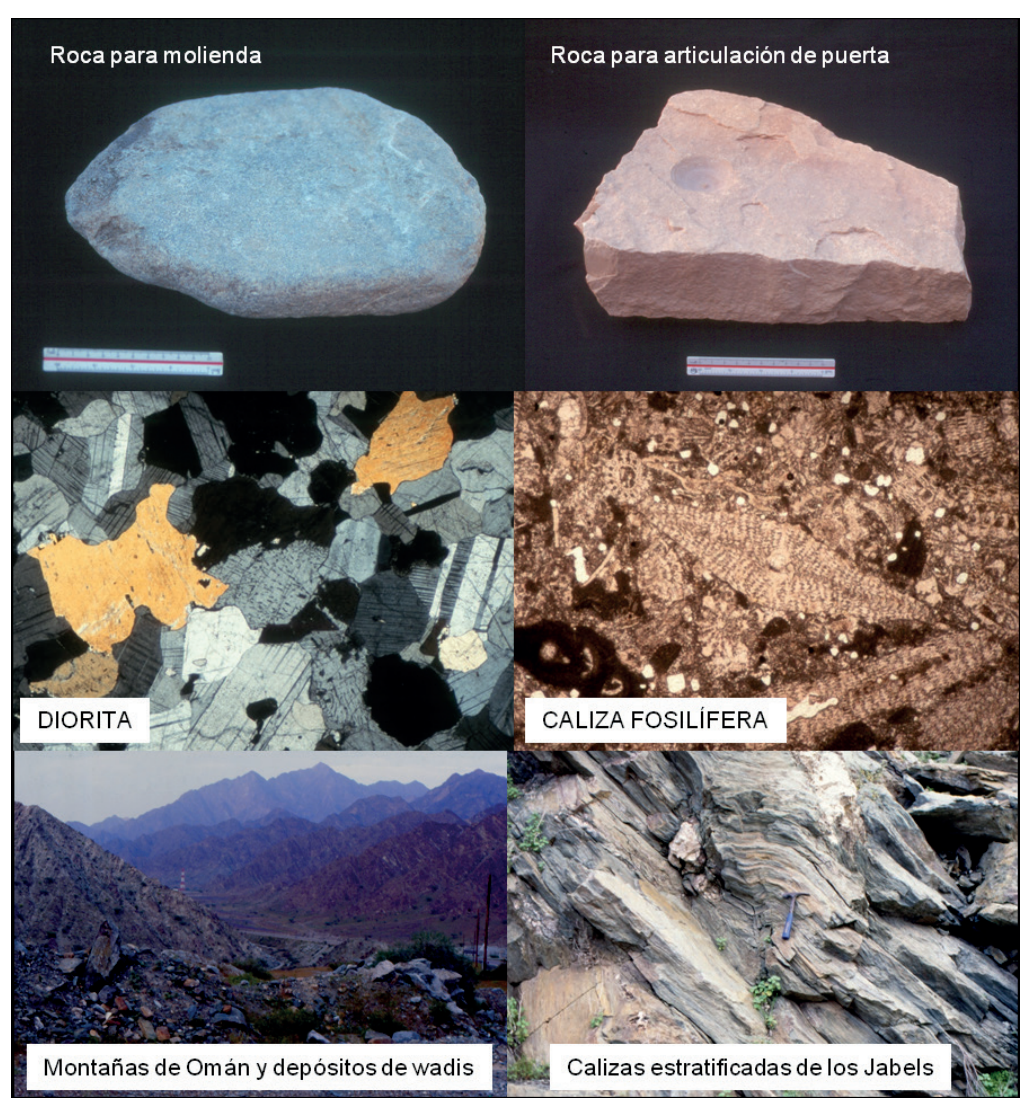

5-Materiales pétreos con evidencias de utilización. Se muestra también la textura de la roca que los forma (petrografía) y el área fuente de estos materiales.

El área de procedencia de las calizas y peridotita podría haber sido el cercano Jabel Buhais. Este anticlinal desventrado muestra en su núcleo afloramientos de rocas peridotíticas, con frecuente fracturación y rellenos de alteración similares a los de la muestra del yacimiento. Por otra parte, las calizas (biomicritas) presentan texturas y fósiles similares a los encontrados en diversos tramos del mencionado cerro. Los útiles de gabro y gabro olivínico parecen tener un área de procedencia más alejada, ya que no abundan en los Jabels próximos. Estas rocas básicas son muy frecuentes en las Montañas de Omán, al este del yacimiento, donde presentan excelentes afloramientos y depósitos con clastos de grano grueso en los wadis.

\section{Vasijas verdes}

Los restos de vasijas elaboradas con rocas blandas ("softstones") están formadas por agregados laminares de clorita presentando colores de verde a negro (Figura 6). La presencia de algunos fragmentos rojizos en los que aparecen fases de alta temperatura como olivino y piroxeno ponen de manifiesto que el material sufrió temperaturas superiores a los $800^{\circ} \mathrm{C}$ (Pozo et al. 2005). El origen de la clorita estudiada es difícil de precisar teniendo en cuenta que este mineral se puede formar en contextos geológicos tan dispares como el diagénetico, el metamórfico o el hidrotermal. Sin embargo, la pureza de la clorita y sus rasgos petrográficos sugieren un origen ligado a la alteración hidrotermal de minerales máficos asociados a rocas básicas o ultrabásicas. La abundante presencia de estas rocas en los complejos ofiolíticos de las Montañas de Omán y en los relativamente cercanos del suroeste de Irán (Tepe Yhaya), apoyaría esta interpretación. 


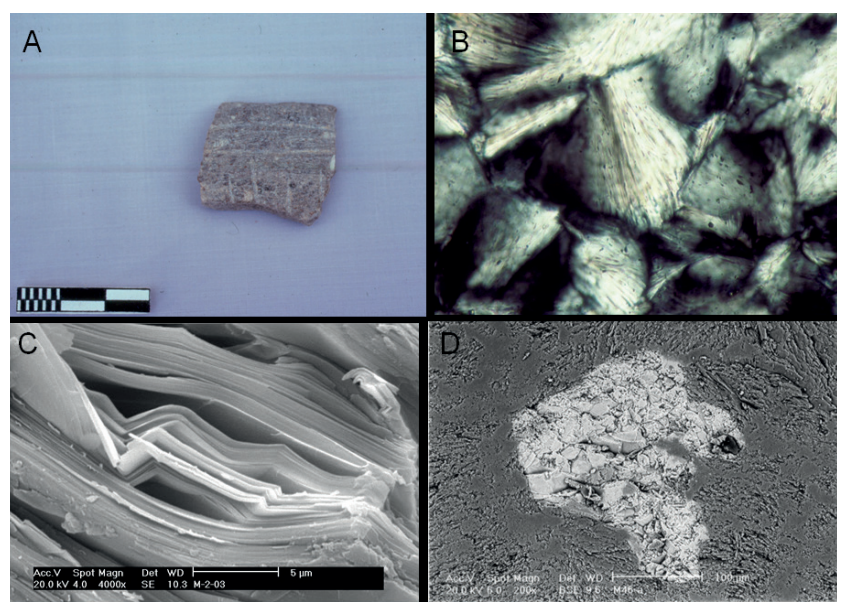

6-Vasijas de clorita. A) fragmento de vasija de roca blanda (soft-stone) de composición cloritica. B) Imagen microscópica (petrografia) donde se observan los agregados fibrorradiales de clorita. C) Microfábrica laminar de la clorita mostrando evidencias de deformación. D) Inclusión de mineral de hierro y titanio (blanco) en la masa de clorita (gris).

III.2. Los yacimientos del valle del río Balih (Siria): materiales cerámicos

La cerámica Halaf adopta su nombre de Tell Halaf, una localidad situada en la cuenca del río Khabur (Siria) un afluente del Éufrates (Figura 7). Se trata de cerámicas pintadas de estilo y textura característica, que constituye una de las pocas mercancías conocidas del denominado período temprano en Mesopotamia, a finales del sexto milenio y parte del quinto (a.C.). A la cultura asociada se denomina Halaf, distribuyéndose sin antecedentes conocidos en la región mesopotámica septentrional (Figura 8A).

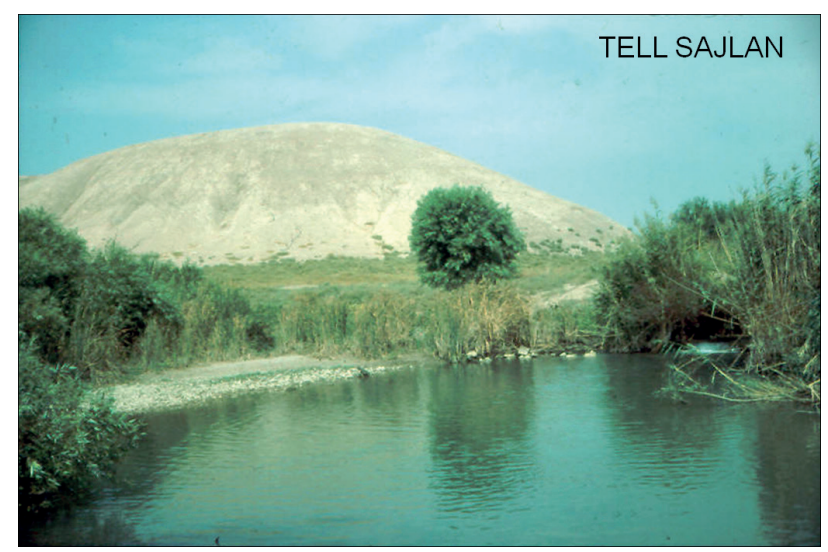

7-Imagen del yacimiento de Tell Sajlan en el valle del Rio Balikh (Siria).

En los tells del valle del rio Balikh son frecuentes las muestras de cerámicas Halaf no estratificadas, que han sido analizadas desde el punto de vista mineralógico, químico y textural (Pozo et al., 1996). Teniendo en cuenta el color se han diferenciado tres tipos de cerámicas: verdes, marrones y rojas. Las técnicas analíticas empleadas incluyen difracción de rayos $\mathrm{X}$, fluorescencia de rayos $\mathrm{X}$ y microscopía óptica.

Desde el punto de vista mineralógico es posible establecer tres asociaciones mineralógicas dependiendo de la proporción de carbonatos, piroxenos o minerales félsicos (cuarzo, feldespatos), lo que implica la utilización de materias primas distintas que corroboran actividad comercial. Considerando la abundancia de calcita o gehlenita, térmicamente antagónicos, junto a la ausencia de wollastonita, la proporción de filosilicatos y la posible neoformación de piroxeno en algunas muestras, permite establecer un rango de 
temperaturas, durante la cocción, entre 700 y $900^{\circ} \mathrm{C}$. Basándose además en la microfábrica (Figura $8 \mathrm{~B}$ y $\mathrm{C}$ ) se ha podido determinar que las cerámicas verdes son las que estuvieron sometidas a una temperatura más alta, y las rojas a la más baja.

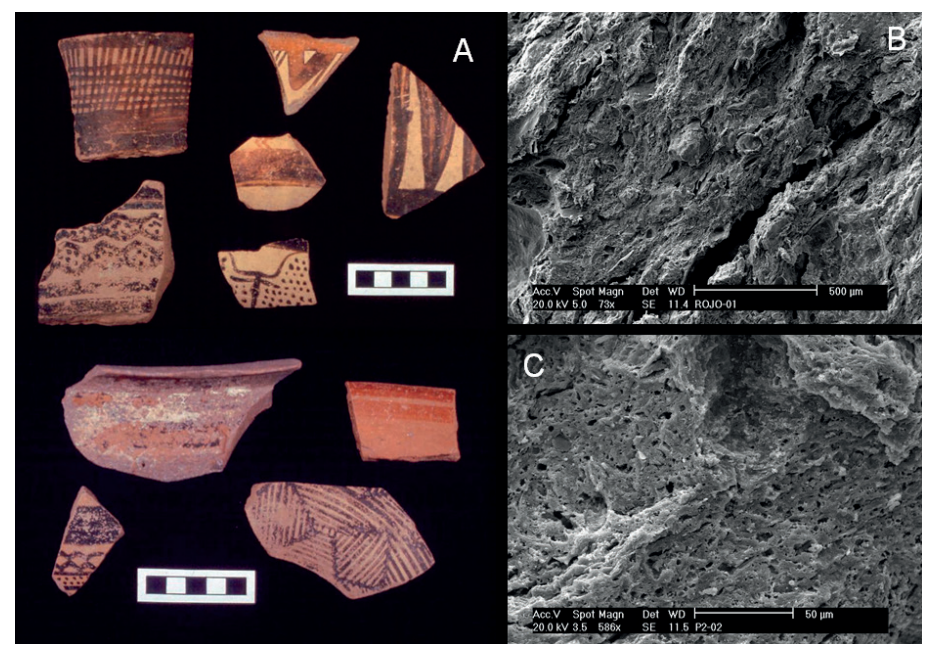

8-Cerámica Halaf. A) Fragmentos cerámicos decorados con pinturas. B) Microfábrica esquelética observada con SEM. C) Microfábrica vacuolar observada con SEM. Las microfábricas se relacionan con el grado de temperatura alcanzado por la cerámica.

La ausencia de correlación entre el color de la cerámica, su posición geográfica y la composición química se ha interpretado como resultado de la coexistencia de pastas locales mezcladas con otras procedentes de la región, lo que indicaría la comercialización a gran escala de estas cerámicas en la Mesopotamia septentrional. La caracterización petrográfica y mineralógica ha resultado especialmente eficaz en el estudio de las materias primas y tratamiento térmico de pastas cerámicas. Los resultados del estudio geoquímico son más limitados, debido a la mezcla de cerámicas con diversa procedencia.

\section{III.3. Saruq al-Hadid: materiales metálicos}

El yacimiento de Saruq al-Hadid se localiza en la zona nororiental del Rub al-Khali, localizado unos $60 \mathrm{~km}$ al sur de la ciudad de Dubai. El yacimiento presenta evidencias de ocupación durante el primer milenio (a.C.), siendo un reconocido asentamiento productor de metales (Casana et al., 2009). Se caracteriza por presentar abundantes restos de escorias silicatadas y metálicas, restos de menas procesadas y lingotes metálicos, junto a una impresionante acumulación de restos arqueológicos que incluyen cerámicas pero especialmente productos diversos fabricados con metales (cobre, bronce, hierro). La ubicación del yacimiento es enigmática ya que se encuentra a una distancia considerable (50-100 km) de cualquier fuente de agua, combustibles (madera) o menas minerales. Aunque en el primer milenio (a.C.) las condiciones ambientales y climáticas permitiesen el abastecimiento de agua y madera, queda el problema del suministro de minerales de cobre que tendría que haber sido transportado desde las Montañas de Omán donde existen evidencias de actividades mineras en la antigüedad (Weeks, 2003). El periodo de ocupación con máxima actividad metalúrgica comenzó alrededor del 1300 a.C. y continúo hasta el 700 a.C. (Herrmann, 2013). 


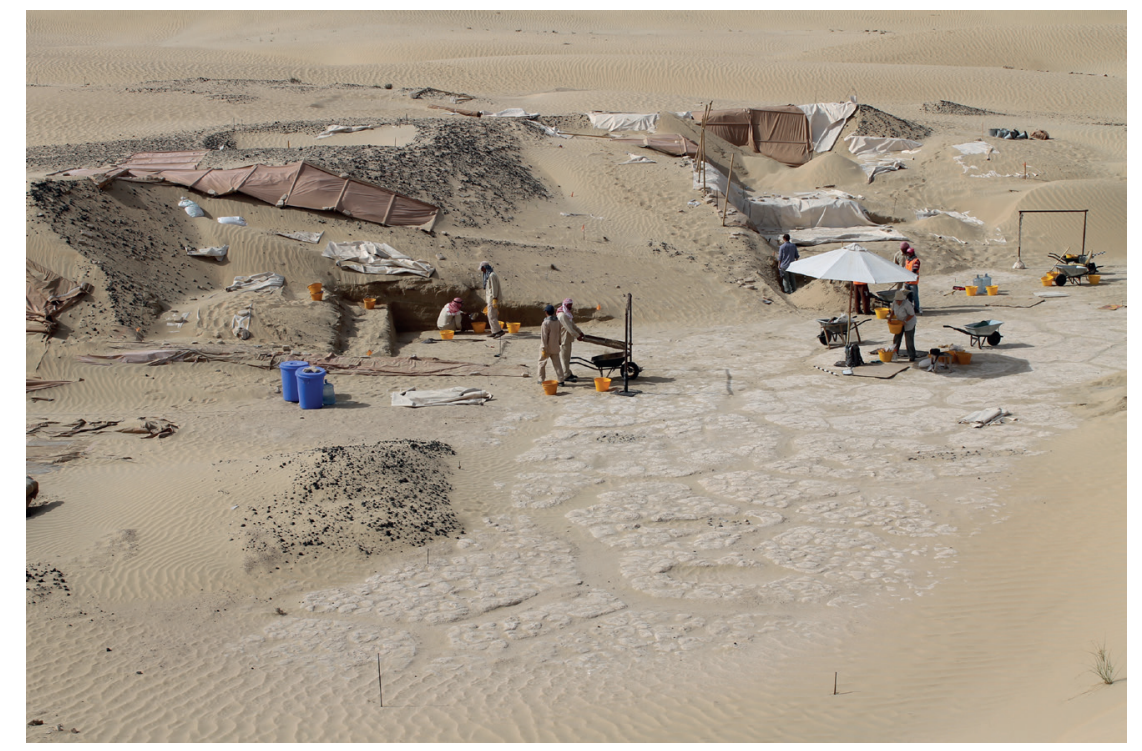

9-Yacimiento de Saruq al-Hadid (Emirato de Dubai). Se observa la presencia de escorias y restos de menas asociados a depósitos eólicos, que se disponen sobre una superficie de composición yesifera.

Los restos arqueológicos y resultantes de la actividad metalúrgica se ubican en diversos niveles de acumulación en depósitos eólicos que tienen como substrato una capa de yesos compactos en los que se ha constatado la evidencia de su utilización para la preparación de cavidades que albergaban los hornos empleados para la obtención de metales, principalmente cobre (Figura 9). En el año 2015 el autor de este trabajo participó en el estudio de materiales resultantes de la actividad metalúrgica con el fin de establecer diferentes tipologías en las escorias y restos de menas y metálicos. A partir de un centenar de muestras se establecieron diversos tipos en función de aspecto, textura y composición mineralógica. Las técnicas mineralógicas empleadas incluyeron: microscopía óptica (lámina delgada y probeta pulida), microscopía electrónica de barrido con análisis puntual EDX y difracción de rayos X (XRD). Todo ello se complementó con el análisis químico mediante fluorescencia de rayos X (XRF), absorción atómica (AA) y análisis de espectroscopia de emisión atómica de plasma acoplado por inducción (ICP-AES).

La determinación de la textura y composición de las muestras demostró ser una excelente herramienta para la clasificación de los diferentes tipos de muestras recogidas en el yacimiento arqueológico, diferenciándose: escorias, restos de menas y lingotes metálicos (Figura 10). Las escorias están formadas por silicatos y óxidos de hierro (hematites o magnetita). En la mayor parte de los casos los silicatos presentan una composición típica ferromagnesiana (olivino, piroxeno), aunque más puntualmente la mineralogía puede ser de tipo félsico (cuarzo, cristobalita y feldespatos) con abundante vidrio. En alguna muestra se han reconocido relictos de la mena metálica o del producto de su alteración (inclusiones verdes). Estos resultados aportan información sobre el tipo de rocas en las que se encontraban las menas minerales, y por lo tanto las características de su yacimiento.

Mezclados con las escorias se observan restos de menas con diverso grado de transformación, que se caracterizan por ser pobres en silicatos pero ricos en hierro y cobre. Dentro de los minerales de cobre se han identificado sulfuros (covellina-calcosina), óxidos (cuprita) y minerales verdes que incluyen carbonatos, cloruros y sulfatos (malaquita, atacamita y brochantita). Con respecto a los restos de menas ricas en hierro los minerales identificados han sido hematites y goethita. La asociación de minerales mena junto a las características de las escorias aporta información sobre su procedencia geográfica dentro del contexto de las Montañas de Omán. 
Un tercer tipo de muestras estudiadas incluye restos de lingotes metálicos en los que a veces quedan restos de escorias y de carbón vegetal. Los principales compuestos metálicos identificados incluyen cobre metálico y óxido de cobre. Sus características aportan información sobre el proceso metalúrgico empleado.

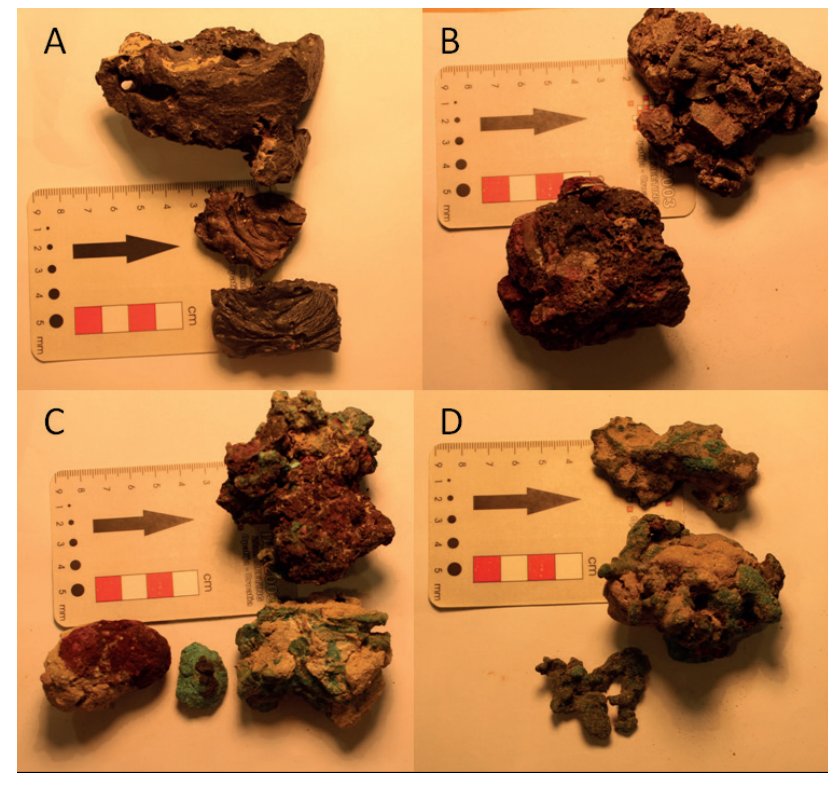

10-Tipologías de materiales metalúrgicos del yacimiento. A) Escoria de composición ultrabásica. B) Escoria de composición félsica. C) Restos de menas parcialmente tratadas metalúrgicamente. D) Lingotes de cobre con recubrimientos verdes de alteración.

Desde el punto de vista químico un hecho relevante es la pobreza en estaño de las muestras estudiadas. Esta escasez se ha observado también en los yacimientos metálicos de las Montañas de Omán. Para explicar el origen de este metal, utilizado con frecuencia en Saruq al-Hadid en la fabricación del bronce, se hace necesaria la existencia de una fuente alóctona, lo que implicaría la existencia de un comercio de intercambio importante.

\section{Consideraciones finales}

Ciencias interdisciplinares como la Geoarqueología y la Arqueometría son un complemento indispensable para la interpretación de los yacimientos arqueológicos y su contenido. Permiten no solo reconstruir el entorno ambiental y geográfico del yacimiento sino también su datación y caracterización de los materiales hallados. El empleo de las técnicas analíticas de caracterización mineralógica y geoquímica, así como el estudio petrográfico son herramientas necesarias para reconstruir e interpretar el pasado y por lo tanto su historia. En muchos casos, junto a otros estudios científicos, es un complemento a la información obtenida de los textos, ayudando a corroborar o descartar algunos de sus contenidos. En el presente trabajo se pone de manifiesto la importancia de conocer la composición (mineralógica y química) y la textura de los diversos materiales (rocas, minerales, sedimentos, cerámicas, metales) que constituyen una parte importante de los hallazgos arqueológicos

\section{Agradecimientos}

Mi agradecimiento al Profesor Dr. Joaquín Córdoba por su generosidad y amistad a lo largo de más de tres décadas de colaboración. Ha sido un verdadero placer compartir este viaje que nos ha transportado a otros momentos del pasado de Oriente. Gracias amigo. El presente trabajo se enmarca dentro de las actividades del Grupo de Investigación UAM GPG-418 (Geomateriales y Procesos Geológicos). 


\section{Referencias}

Butzer, K.W., 1982, Archaeology as human ecology. Cambridge University Press. Cambridge.

Canti, M., 2001, "What is geoarchaeology? Re-examining the relationship between archaeology and Earth sciences", in: Albarella, U. (ed.), Environmental Archaeology: Meaning and purposes, Environmental Science and Technology Library, Vol. 17, Springer, Dordrecht. Pp. 103-112.

Casana, J., Herrmann, J.T., Qandil, H.S, 2009, "Settlement history in the Eartern Rub al-Khali: Preliminary report of the Dubai desert survey (2006-2007)", Arabian Archaeology and Epigraphy, 20, Blackwell Publishing Ldt, pp. 30-45.

Gladfelter, M., 1977, "Geoarchaeology: The geomorphologist and Archaeology", American Antiquity. 42, pp. 519-538.

Hassan, F.A., 1979, "Geoarchaeology: The geologist and Archaeology", American Antiquity. 44, pp. 267-270.

Herrmann, J.T., 2013, “Three dimensional mapping of archaeological and sedimentary deposits with Ground-penetrating Radar at Saruq al-Hadid, Dubai, United Arab Emirates”, Archaeological Prospection 20, pp. 189-203. Wiley.

North, F.J., 1938, "Geology for archaeologists”. Archaeological Journal 94, pp. 73115.

Pozo, M., 1998, "Contribución de la investigación geológica en la resolución de problemas arqueológicos de las culturas antiguas", Arbor, vol. CLXI, pp. 293-310.

Pozo, M., Córdoba, J., 2002, “Architecture, implements and geological constraints. Provenance study and archaeology on the uses and technologies of an iron age village (AM.2-Thuqueibah, Emirate of Sharjah, UAE)", Proceedings of the Seminar for Arabian Studies 32, pp. 63-74.

Pozo, M., Casas, J., Moreno, A., Martín, J.A., Medina, J.A., 1996, "Estudio mineralógico, textural y químico de restos de cerámica Halaf en el norte del valle Balih (norte de Siria)", Boletín de la Sociedad Española de Mineralogía 19, pp. 85-99.

Pozo, M., Casas, J., Medina, J.A., Martín Rubí, J.A., 2005, "Estudio composicional de rocas blandas (soft-stones) procedentes de vasijas del yacimiento arqueológico de Jhebel Buhays (Emirato Árabe Unido de Sharjah). Importancia del factor textural", Boletín Geológico y Minero 116, pp. 39-52.

Pozo, M., Casas, J., Moreno, A., Martín-Rubí, J.A.,1998, “Aplicación de técnicas geológicas al estudio de fragmentos de cerámica Halaf de la cuenca del río Balikh (Siria)", Isimu, vol.1, pp. 296-308.

Pozo, M., Casas, J., Martín Rubí, J.A., 1999, "Estudio mineralógico, químico y textural de materiales y elementos arqueológicos en asentamientos de la Edad del Hierro del Oasis de Al-Madam. (Emirato de Sharjah, EE.AA.UU)". Isimu 2, pp. 605-634.

Pozo, M., González, J., Giner, J., 2004, Geología práctica. Introducción al reconocimiento de materiales y análisis de mapas. Pearson Prentice Hall. Madrid.

Weeks, L.R., 2003, Early metallurgy of the Persian Gulf; Technology, trade and the Bronze Age World. Brill. 